Author affiliations and support information (if applicable) appear at the end of this article.

Published at jco.org on August 28, 2017 Corresponding author: Carmen Martínez, MD, Hematology Department, Institute of Hematology and Oncology, Hospital Clínic, Villarroel 170, Barcelona, 08036 Spain; e-mail: cmarti@clinic.ub.es. () 2017 by American Society of Clinical Oncology

0732-183X/17/3530w-3425w/\$20.00
ASSOCIATED CONTENT

(2) Appendix

DOI: https://doi.org/10.1200/JCO 2017.72.6869

\section{Post-Transplantation Cyclophosphamide-Based Haploidentical Transplantation as Alternative to Matched Sibling or Unrelated Donor Transplantation for Hodgkin Lymphoma: A Registry Study of the Lymphoma Working Party of the European Society for Blood and Marrow Transplantation}

Carmen Martínez, Jorge Gayoso, Carmen Canals, Hervé Finel, Karl Peggs, Alida Dominietto, Luca Castagna, Boris Afanasyev, Stephen Robinson, Didier Blaise, Paolo Corradini, Maija Itälä-Remes, Arancha Bermúdez, Edouard Forcade, Domenico Russo, Michael Potter, Grant McQuaker, Ibrahim Yakoub-Agha, Christof Scheid, Adrian Bloor, Silvia Montoto, Peter Dreger, and Anna Sureda on behalf of the Lymphoma Working Party of the European Group for Blood and Marrow Transplantation

\section{$\begin{array}{llllllll}\text { A } & \text { B } & \mathbf{S} & \mathbf{T} & \mathbf{R} & \mathbf{A} & \mathbf{C} & \mathbf{T}\end{array}$}

\section{Purpose}

To compare the outcome of patients with Hodgkin lymphoma who received post-transplantation cyclophosphamide-based haploidentical (HAPLO) allogeneic hematopoietic cell transplantation with the outcome of patients who received conventional HLA-matched sibling donor (SIB) and HLAmatched unrelated donor (MUD).

\section{Patients and Methods}

We retrospectively evaluated 709 adult patients with Hodgkin lymphoma who were registered in the European Society for Blood and Marrow Transplantation database who received HAPLO ( $n=98$ ), SIB ( $n=338)$, or MUD ( $n=273$ ) transplantation.

\section{Results}

Median follow-up of survivors was 29 months. No differences were observed between groups in the incidence of acute graft-versus-host disease (GVHD). HAPLO was associated with a lower risk of chronic GVHD (26\%) compared with MUD (41\%; $P=.04)$. Cumulative incidence of nonrelapse mortality at 1 year was 17\%, 13\%, and 21\% in HAPLO, SIB, and MUD, respectively, and corresponding 2-year cumulative incidence of relapse or progression was 39\%, 49\%, and $32 \%$, respectively. On multivariable analysis, relative to SIB, nonrelapse mortality was similar in HAPLO $(P=.26)$ and higher in MUD $(P=.003)$, and risk of relapse was lower in both HAPLO $(P=.047)$ and MUD $(P<.001)$. Two-year overall survival and progression-free survival were $67 \%$ and $43 \%$ for HAPLO, $71 \%$ and $38 \%$ for SIB, and $62 \%$ and $45 \%$ for MUD, respectively. There were no significant differences in overall survival or progression-free survival between HAPLO and SIB or MUD. The rate of the composite end point of extensive chronic GVHD and relapse-free survival was significantly better for HAPLO $(40 \%)$ compared with SIB $(28 \% ; P=.049)$ and similar to MUD (38\%; $P=.59)$.

\section{Conclusion}

Post-transplantation cyclophosphamide-based HAPLO transplantation results in similar survival outcomes compared with SIB and MUD, which confirms its suitability when no conventional donor is available. Our results also suggest that HAPLO results in a lower risk of chronic GVHD than MUD transplantation.

J Clin Oncol 35:3425-3432. (C) 2017 by American Society of Clinical Oncology 
INTRODUCTION

The number of patients with Hodgkin lymphoma (HL) who are treated with allogeneic hematopoietic cell transplantation (alloHCT) has significantly increased over the last decade. ${ }^{1,2}$ Several retrospective studies and one prospective clinical trial have demonstrated that young patients with chemosensitive disease can clearly benefit from alloHCT using reduced-intensity conditioning (RIC) regimens. ${ }^{3-9}$ According to the current recommendations of the European Society for Blood and Marrow Transplantation (EBMT), alloHCT is considered the standard treatment option in eligible patients who experience sensitive relapse after autologous hematopoietic cell transplantation and with a sibling (SIB) or a matched unrelated (MUD) donor ${ }^{2}$; however, there is a significant proportion of patients for whom a conventional donor is not available. To address this issue, other options, such as HLA-haploidentical transplantation (HAPLO) or cord blood transplantation, have been explored.

HAPLO transplantation with extensive in vivo or ex vivo T-cell depletion that has been used to reduce the risk of severe graft-versushost disease (GVHD) was historically associated with poor outcome, mainly because of a high risk of nonrelapse mortality $(\mathrm{NRM})$, disease relapse, and delayed immune reconstitution ${ }^{10,11}$; however, encouraging results have been reported with HAPLO after a nonmyeloablative regimen and post-transplantation cyclophosphamide (PTCy) as GVHD prophylaxis. ${ }^{12-18}$ Three recent registry studies (two from the Center for International Blood and Marrow Transplant Research and another from EBMT, including patients with lymphoid malignancies) suggest similar survival outcomes with lower incidence of chronic GVHD for HAPLO using PTCy compared with SIB and MUD transplantations. ${ }^{18-20}$

NRM, disease relapse, survival, and GVHD incidence have been historically recognized as the main single end points with which to evaluate the effectiveness of novel alloHCT strategies; however, as a result of the complexity of the alloHCT procedure in which decreases in NRM and GVHD often come at the cost of increased relapse, no one factor is sufficient when examining post-transplantation outcomes. Recently, novel composite end points that encompass not only mortality and relapse, but other clinically meaningful post-transplant events, such as GVHD, are being increasingly used to quantify survival without significant morbidity after alloHCT. ${ }^{21-24}$ GVHD-free/relapse-free survival (GRFS) has developed as a novel composite end point that may be more indicative of clinical success when comparing traditional alloHCT procedures with new platforms, such as HAPLO using PTCy.

The objective of the current study was to compare the outcomes of alloHCT, including GRFS, using HAPLO donors with the PTCy approach with the outcomes of conventional HLA-identical SIB donors and MUD donors for patients with HL.

\section{PATIENTS AND METHODS}

\section{Data Source}

EBMT is a voluntary organization that comprises more than 500 transplant centers, mainly from Europe. Accreditation as a member center requires submission of the minimal essential data form A from all consecutive patients to a central registry from which patients can be identified by diagnosis of underlying disease and type of transplantation. Minimal essential data form A data are annually updated. Informed consent for transplantation and data collection was obtained locally according to regulations that were applicable at the time of transplantation. Since January 1, 2003, all transplantation centers have been required to obtain written informed consent before data registration with the EBMT in accordance with the 1975 Helsinki Declaration.

\section{Patient Eligibility}

Eligible patients were age $\geq 18$ years and had undergone alloHCT for HL between January 2010 and December 2013. Transplantations from mismatched related donors - two or more mismatches-were identified as haploidentical transplantations. Baseline information and transplantation characteristics of eligible patients were downloaded and centers were contacted to provide additional information about post-transplant outcomes. Outcomes of patients who received PTCy-based HAPLO was compared with those of patients who were identified in the EBMT database as having received transplantation from a matched SIB donor or MUD (matched at the allele-level at HLA-A, -B, -C, and -DRB1) but otherwise meeting the eligibility criteria described above. Patients who received ex vivo graft manipulation were not eligible.

\section{Statistical Analysis}

Patient characteristics were compared by using a Kruskall-Wallis test for quantitative variables, and $\chi^{2}$ or Fisher's exact test for categorical variables. NRM was defined as the time from alloHCT to death in the absence of prior relapse and/or progression. Relapse rate was calculated as the time from alloHCT to relapse and/or progression. NRM and relapse rate events were considered as competing risks. Chronic GVHD (cGVHD) was also analyzed in a competing-risks setting, with death and relapse as competing events. Progression-free survival (PFS) was defined as the time from alloHCT to relapse and/or progression or death from any cause, and overall survival (OS) was defined as the time from alloHCT to death from any cause. A composite end point defined as extensive cGVHD-free, relapse-free survival (cGRFS) after transplantation was also studied. This end point was calculated as the time from alloHCT to relapse and/or progression, to the onset of extensive cGVHD, or to death from any cause.

Probabilities of OS, PFS, and cGRFS were estimated by using the Kaplan-Meier product limit method and compared by using the log-rank test. Estimates of NRM, relapse rate, and cGVHD were calculated by using cumulative incidence curves to accommodate competing risks, and were compared by using Gray's test. The effect of donor type on NRM, relapse rate, PFS, and OS was assessed by multivariable analyses (Cox proportional hazards regression models or competing risk proportional subdistribution hazards regression models) adjusting by potential prognostic factors. The effect of donor type was investigated by multivariable models, adjusting for time from HL diagnosis to alloHCT, sex, age at transplantation, previous autologous hematopoietic cell transplantation, disease status at transplantation, performance status at transplantation, type of conditioning regimen, patient and donor cytomegalovirus status, and donor sex. Several models were constructed that explored different cutoff values for continuous variables or classifying disease status in different groups or in two categories (sensitive $v$ refractory disease). The proportionality assumption was tested for each covariate factor. All variables met the proportionality assumption. A backward stepwise method was used that included the type of donor as the main factor-of-interest in all steps of model building. Survival and cumulative incidence results are presented as estimates and 95\% CIs. Association of factors with end points is presented as relative risk (RR) and $95 \%$ CI. All tests were two-sided and $P$ values $<.05$ were considered as indicating significant associations. Analyses were performed by using the SPSS for Windows version 20.0 (SPSS, Chicago, IL) and R Project software, version 2.15.2. 


\section{RESULTS}

\section{Baseline Characteristics}

Baseline patient-, disease- and transplantation-related characteristics are listed in Table 1. A significantly higher number of patients in the HAPLO group received RIC regimens, bone marrow stem-cells (BMSC), and had female donors. Patients in the SIB and MUD groups received calcineurin inhibitor-based prophylaxis plus antithymocyte globulin (ATG; 14\% and 51\%, respectively) or campath (23\% and $23 \%$, respectively).

\section{Hematopoietic Recovery}

The cumulative incidence of neutrophil recovery at day 28 for patients who received BMSC was $96 \%$ (95\% CI, 91 to 100) in the HAPLO group compared with $94 \%$ (95\% CI, 86 to 100) and 93\% (95\% CI, 84 to 100) in the SIB and MUD groups, respectively $(P=.82)$. The day 28 cumulative incidence of platelet recovery in similar order was $67 \%$ (95\% CI, 48 to 92 ), $87 \%$ (95\% CI, 75 to $100)$, and $79 \%$ (95\% CI, 63 to 99) $(P=.79)$. For peripheral blood stem-cell (PBSC) transplantations, the cumulative incidence of neutrophil recovery was $97 \%$ (95\% CI, 92 to 100) in the HAPLO group compared with $96 \%$ (95\% CI, 94 to 98 ) and 94\% (95\% CI, 91 to 97$)$ in the SIB and MUD groups, respectively $(P=.02)$. The cumulative incidence of platelet recovery in similar order was $72 \%$ (95\% CI, 54 to 96), 90\% (95\% CI, 85 to 95), and 89\% (95\% CI, 84 to $95 ; P=.01)$. Engraftment results expressed as median time and ranges to recovery are provided in the Appendix (online only).

\section{Acute and cGVHD}

The rate of grade II to IV acute GVHD after HAPLO was higher compared with that of SIB $(33 \% \vee 18 \% ; P=.003)$, whereas no differences were observed in comparison with MUD ( $30 \% ; P=.36)$. The incidence of grade III to IV acute GVHD was similar between the three groups (HAPLO, 9\%; SIB, 6\%; and MUD, $9 \% ; P=.54)$. The cumulative incidence of cGVHD at 1 year after HAPLO, SIB, and MUD was 26\% (95\% CI, 18 to 39), $25 \%$ (95\% CI, 20 to 32 ), and $41 \%$ (95\% CI, 34 to 48$)$, respectively $(P=.017)$. In univariable analysis, MUD was associated with a higher risk of cGVHD compared with SIB $(P=.012)$ and HAPLO $(P=.04$; Table 2 and Fig 1$)$. Results were

\begin{tabular}{|c|c|c|c|c|}
\hline Characteristic & $\begin{array}{l}\text { HAPLO } \\
(n=98)\end{array}$ & $\begin{array}{c}\text { SIB } \\
(n=338)\end{array}$ & $\begin{array}{c}\text { MUD } \\
(n=273)\end{array}$ & $P$ \\
\hline Median age at alloHCT, years (range) & $31(18-68)$ & $32(18-67)$ & $32(18-68)$ & .37 \\
\hline Sex, No. $(\%)$ & & & & .18 \\
\hline Male & $56(57)$ & $193(57)$ & $175(64)$ & \\
\hline Female & $42(43)$ & $145(43)$ & $98(36)$ & \\
\hline Performance status, No. (\%) & & & & .84 \\
\hline Good (> 80\% KS) & $81(95)$ & 301 (95) & $240(96)$ & \\
\hline Poor (<80\% KS) & $4(5)$ & $16(5)$ & $10(4)$ & \\
\hline HL status, No. (\%) & & & & .1 \\
\hline Chemosensitive & $83(85)$ & $264(78)$ & $230(84)$ & \\
\hline Refractory & $15(15)$ & $74(22)$ & $43(16)$ & \\
\hline Yes for previous autoHCT, No. (\%) & 75 (77) & $236(70)$ & $206(76)$ & .2 \\
\hline Median time from $\mathrm{HL}$ diagnosis to alloHCT, months (range) & $30(11-179)$ & $28(6-343)$ & 35 (3-269) & .01 \\
\hline Source of stem cells, No. (\%) & & & & $<.001$ \\
\hline Bone marrow & $60(61)$ & $33(10)$ & $31(11)$ & \\
\hline Peripheral blood & 38 (39) & $302(89)$ & $241(88)$ & \\
\hline Female donor, No. (\%) & $54(55)$ & $152(45)$ & $61(23)$ & $<.001$ \\
\hline Reduced intensity conditioning regimen, No. (\%) & $84(90)$ & $218(69)$ & $172(69)$ & $<.001$ \\
\hline $\mathrm{TBI}$ in conditioning, No. (\%) & $61(62)$ & $42(13)$ & $28(10)$ & $<.001$ \\
\hline Conditioning regimen, No. (\%) & & & & $<.001$ \\
\hline Flu +Cy + 2 Gy TBI & $57(58)$ & $4(1)$ & $3(1)$ & \\
\hline $\mathrm{Flu}+\mathrm{Cy}+\mathrm{Bu}$ & $22(22)$ & $1(0.3)$ & 0 & \\
\hline $\mathrm{Flu}+\mathrm{Mel}$ & $2(2)$ & 112 (33) & $82(30)$ & \\
\hline $\mathrm{Flu}+\mathrm{Bu}$ & $2(2)$ & $51(15)$ & $41(15)$ & \\
\hline Other Flu-based regimen & $10(10)$ & $88(26)$ & $73(27)$ & \\
\hline BEAM & 0 & $36(11)$ & $28(10)$ & \\
\hline $\mathrm{Cy}+\mathrm{TBI}$ or $\mathrm{Bu}$ & $1(1)$ & $20(6)$ & $9(3)$ & \\
\hline Other & $4(4)$ & $26(8)$ & $37(13)$ & \\
\hline GVHD prophylaxis, No. (\%) & & & & $<.001$ \\
\hline Post-transplantation Cy & $98(100)$ & - & - & \\
\hline $\mathrm{CNI}+\mathrm{MTX} \pm$ others & $2(2)$ & $132(39)$ & $90(33)$ & \\
\hline $\mathrm{CNI}+\mathrm{MMF} \pm$ others (except MTX) & 92 (94) & $90(27)$ & 90 (34) & \\
\hline $\mathrm{CNI} \pm$ others (except MTX/MMF) & 0 & $88(26)$ & $64(23)$ & \\
\hline ATG as part of GVHD profilaxis & 0 & $48(14)$ & $139(51)$ & \\
\hline Campath as part of GVHD profilaxis & 0 & $79(23)$ & $64(23)$ & \\
\hline
\end{tabular}




\begin{tabular}{|c|c|c|c|c|}
\hline Outcomes & HAPLO $(n=98)$ & $\operatorname{SIB}(n=338)$ & MUD $(n=273)$ & $P$ \\
\hline NRM & & & & .023 \\
\hline 1-year cumulative incidence & $17 \%$ & $13 \%$ & $21 \%$ & HAPLO $v$ SIB, .37 \\
\hline $95 \% \mathrm{Cl}$ & 11 to 27 & 10 to 17 & 16 to 26 & $\begin{array}{l}\text { HAPLO } v \text { MUD, } .30 \\
\text { SIB } v \text { MUD, .006 }\end{array}$ \\
\hline Relapse rate & & & & $<.001$ \\
\hline 2-year cumulative incidence & $39 \%$ & $49 \%$ & $32 \%$ & HAPLO $v$ SIB, .039 \\
\hline $95 \% \mathrm{Cl}$ & 30 to 51 & 43 to 55 & 26 to 38 & $\begin{array}{l}\text { HAPLO } v \text { MUD, } .31 \\
\text { SIB } v \text { MUD, }<.001\end{array}$ \\
\hline PFS & & & & .086 \\
\hline 2-year cumulative incidence & $43 \%$ & $38 \%$ & $45 \%$ & HAPLO $v$ SIB, .18 \\
\hline $95 \% \mathrm{Cl}$ & 33 to 54 & 31 to 43 & 39 to 51 & $\begin{array}{l}\text { HAPLO } v \text { MUD, } .91 \\
\text { SIB } v \text { MUD, } .038\end{array}$ \\
\hline OS & & & & .118 \\
\hline 2-year cumulative incidence & $67 \%$ & $71 \%$ & $62 \%$ & HAPLO $v$ SIB, .51 \\
\hline $95 \% \mathrm{Cl}$ & 57 to 76 & 66 to 76 & 56 to 68 & $\begin{array}{l}\text { HAPLO } v \text { MUD, } .44 \\
\text { SIB } v \text { MUD, } .039\end{array}$ \\
\hline Chronic GVHD & & & & .017 \\
\hline 1-year cumulative incidence & $26 \%$ & $25 \%$ & $41 \%$ & HAPLO $v$ SIB, .90 \\
\hline $95 \% \mathrm{Cl}$ & 18 to 39 & 20 to 32 & 34 to 49 & $\begin{array}{l}\text { HAPLO } v \text { MUD, } .040 \\
\text { SIB } v \text { MUD, } .012\end{array}$ \\
\hline Extensive cGVHD and PFS & & & & .04 \\
\hline 2-year cumulative incidence & $40 \%$ & $28 \%$ & $38 \%$ & HAPLO $v$ SIB, .049 \\
\hline \multirow[t]{2}{*}{$95 \% \mathrm{Cl}$} & 29 to 51 & 23 to 34 & 32 to 44 & HAPLO $v$ MUD, .59 \\
\hline & & & & SIB $v$ MUD, .038 \\
\hline
\end{tabular}

similar when we analyzed the cumulative incidence of cGVHD according to BMSC or PBSC.

\section{NRM and Relapse}

One hundred twenty-nine (18\%) patients died without experiencing disease relapse and/or progression. No differences in the cumulative incidence of NRM at 1 year were observed between HAPLO and SIB (17\% $v 13 \% ; P=.37)$, whereas MUD had a significantly higher NRM compared with SIB (21\% v 13\%; $P=.006$; Table 2 and Fig 2A). In multivariable analysis, the variables associated with a higher NRM were MUD (RR, 1.78; 95\% CI, 1.21 to $2.60 ; P=.003$ ), age $\geq 40$ years (RR, $1.78 ; 95 \% \mathrm{CI}, 1.24$ to $2.55 ; P=.002$ ), poor performance status (RR, $2.39 ; 95 \% \mathrm{CI}, 1.22$ to $1.68 ; P=.011)$, and refractory HL (RR, $1.62 ; 95 \% \mathrm{CI}, 1.05$ to 2.50; $P=.03$; Table 3$)$.

A total of $280(39 \%)$ patients experienced relapse. The cumulative incidence of disease progression and/or relapse at 2 years was $39 \%$ (95\% CI, 30 to 51), $49 \%$ (95\% CI, 43 to 55), and $32 \%$ (95\% CI, 26 to 38) after HAPLO, SIB, and MUD, respectively (Fig 2B). In multivariable analysis, SIB was associated with a higher relapse risk compared with MUD (RR, 0.57; 95\% CI, 0.43 to 0.74; $P<.001)$ and HAPLO (RR, 0.69; 95\% CI, 0.48 to 0.99; $P=.047$; Table 3). Other factors associated with a higher relapse rate were male gender (RR, $1.28 ; 95 \% \mathrm{CI}, 1.01$ to $1.63 ; P=.049)$, poor performance status (RR, $1.88 ; 95 \% \mathrm{CI}, 1.1$ to $3.2 ; P=.02$ ), and refractory $\mathrm{HL}$ (RR, 2.67; 95\% CI, 2.04 to 3.48; $P<.001$; Table 3 ).

\section{Survival}

Four hundred forty-eight (63\%) of 709 patients are currently alive, with a median follow-up for surviving patients of 27 months (range, 1.1 to 64 months) for HAPLO, 27 months (range, 1 to 76 months) for SIB, and 31 months (range, 0.9 to 70 months) for MUD. The 2-year OS was not significantly different between HAPLO (67\%; 95\% CI, 57 to 76) and SIB (71\%; 95\% CI, 66 to

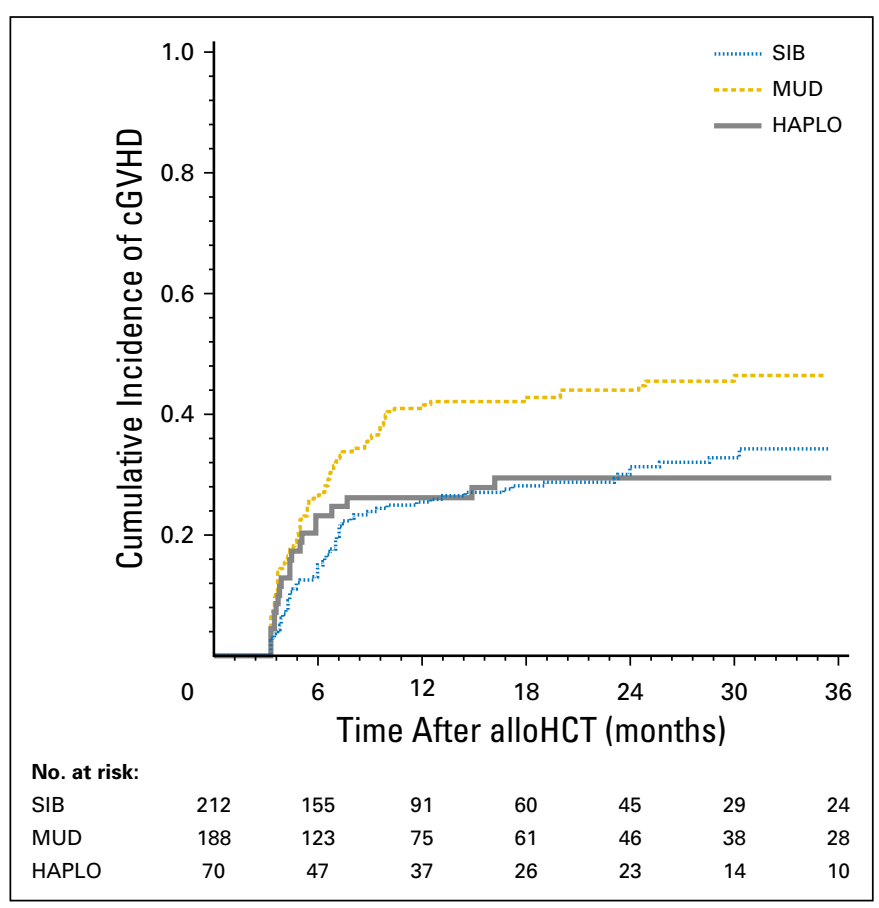

Fig 1. Cumulative incidence of chronic graft-versus-host-disease (cGVHD; overall, $P=.017$ ). alloHCT, allogeneic hematopoietic stem cell transplantation; HAPLO, haploidentical donor; MUD, match-unrelated donor; SIB, sibling donor. 


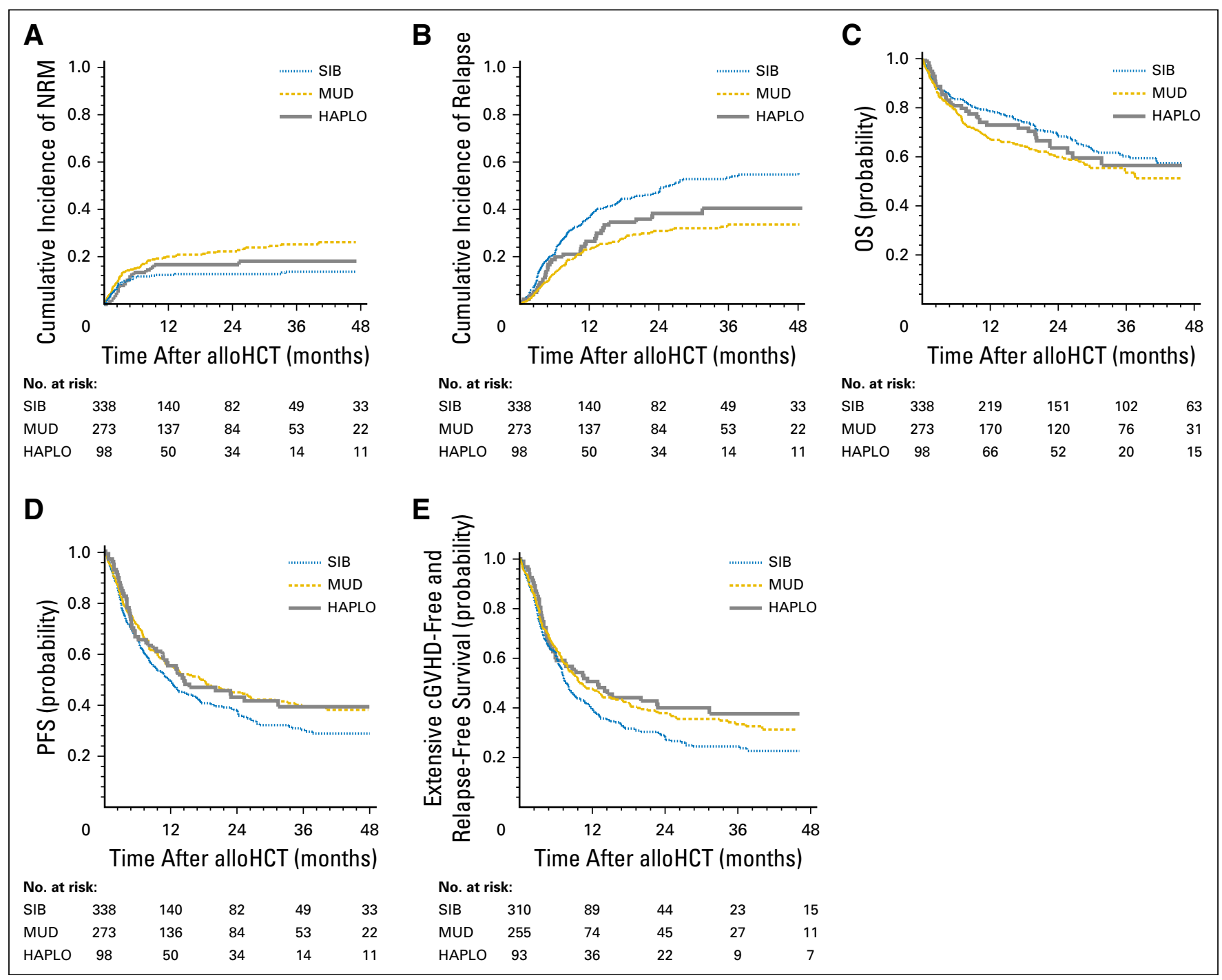

Fig 2. (A) Cumulative incidence of nonrelapse mortality (NRM) in recipients of sibling donor (SIB), match-unrelated donor (MUD), and haploidentical donor (HAPLO) transplantations (overall, $P=.23$ ). (B) Cumulative incidence of relapse and/or progression in recipients of SIB, MUD, and HAPLO transplantations (overall, $P<.001$ ). (C) Kaplan-Meier estimate of overall survival (OS) in recipients of SIB, MUD, and HAPLO transplantations (overall, $P=.118$ ). (D) Kaplan-Meier estimate of progression-free survival (PFS) in recipients of SIB, MUD, and HAPLO transplantations (overall, $P=.086$ ). (E) Kaplan-Meier estimate of combined incidence of extensive chronic graft-versus-host-disease (cGVHD)-free and relapse-free survival in recipients of SIB, MUD, and HAPLO transplantations (overall, $P=.04$ ).

76; $P=.51)$, whereas patients who underwent MUD transplantation had a lower OS $(62 \% ; 95 \% \mathrm{CI}, 56$ to $68 ; P=.039)$ than those who received an SIB alloHCT (Fig 2C). These results were confirmed in multivariable analysis (Table 3). Other predictors for a worse OS were age $\geq 40$ years (RR, $1.62 ; 95 \% \mathrm{CI}$, 1.25 to $2.11 ; P<.001$ ), poor performance status (RR, $2.4 ; 95 \%$ CI, 1.51 to $3.83 ; P<.001)$, and refractory $\mathrm{HL}(\mathrm{RR}, 2.15 ; 95 \% \mathrm{CI}$, 1.62 to $2.85 ; P<.001$; Table 3 ).

The 2-year PFS was similar between HAPLO and SIB (43\%; 95\% CI, 33 to 54 ; and $38 \%$; 95\% CI, 31 to 43 , respectively; $P .18)$, but significantly better after MUD (45\%; $95 \%$ CI, 39 to $51 ; P=.038$ ) than after an SIB alloHCT (Fig 2D). Multivariable analysis did not demonstrate significant differences in PFS after HAPLO or MUD in relation to SIB (Table 3 ). Factors associated with a worse PFS were age $\geq 40$ years (RR, $1.31 ; 95 \% \mathrm{CI}, 1.06$ to $1.63 ; P=.014)$, poor performance status (RR, 2.05; 95\% CI, 1.35 to $3.11 ; P=.001$ ), and refractory HL (RR, 2.22; 95\% CI, 1.76 to $2.79 ; P<.001)$. The rate of the composite end point of 2 -year cGRFS was significantly better for HAPLO ( $40 \%$; $95 \%$ CI, 29 to 51 ) compared with SIB (28\%; 95\% CI, 23 to 34; $P=.049)$ and similar to MUD (38\%; 95\% CI, 32 to $44 ; P=.59$; Fig $2 \mathrm{E}$ ).

A multivariable subanalysis that included HAPLO, SIB, MUD with ATG, and MUD without ATG indicated that HAPLO was associated with a lower relapse rate than SIB (hazard ratio, 0.69 ; $95 \% \mathrm{CI}, 0.48$ to $1.0 ; P=.047)$ with no significant differences in NRM, OS, or PFS. No differences were observed between HAPLO and MUD with or without ATG (Appendix and Appendix Table A1, online only). These results were in line with the outcomes shown in the main analysis. 
Table 3. Multivariable Cox Proportional Hazards Models With Donor Type as a Covariate

\begin{tabular}{cccc}
\hline Factor & $\mathrm{HR}$ & $95 \% \mathrm{Cl}$ & $P$ \\
\hline
\end{tabular}

\section{Cox model on NRM}

Donor type

SIB

MUD

HAPLO

1.78

1.38

1.21 to 2.60

0.79 to 2.39

Age, years

$\leq 39$

$\geq 40$

1.78

1.24 to 2.55

.002

Performance status

Good

Poor

2.39

1.22 to 4.68

.011

Refractory HL

Nonrefractory

Refractory

1.62

1.05 to 2.50

.03

Cox model on relapse rate

Donor type

SIB

MUD

HAPLO

0.57

0.69

0.43 to 0.74

0.48 to 0.99

$<.001$

Sex

Female

Male

Performance status

Good

Poor

Refractory $\mathrm{HL}$

Nonrefractory

Refractory

Cox model on OS

Donor type

SIB

MUD

HAPLO

Age, years

$\leq 39$

$\geq 40$

1.28

1.01 to 1.63

.049

Performance status

Good

Poor

Refractory HL

Nonrefractory

Refractory

Cox model on PFS

Donor type

SIB

MUD

HAPLO

Age, years

$\leq 39$

$\geq 40$

Performance status

Good

Poor

\subsection{8}

1.1 to 3.2

.02

2.04 to 3.48

Refractory HL

Nonrefractory

Refractory

1.47

1.13 to 1.92

0.84 to 1.82

Abbreviations: HAPLO, haploidentical donor; $\mathrm{HL}$, Hodgkin lymphoma; $\mathrm{HR}$ hazard ratio; MUD, match-unrelated donor; NRM, nonrelapse mortality; OS, overall survival; PFS, progression-free survival; SIB, sibling donor.

\section{DISCUSSION}

Here, we describe the largest series of patients with HL who underwent HAPLO alloHCT that used PTCy-based GVHD prophylaxis compared with transplantations that used either a MUD or an SIB donor. This registry analysis demonstrates that the survival outcomes of HAPLO transplantation are comparable with that of conventional SIB and MUD transplantations across multiple centers and conditioning regimens. Moreover, HAPLO alloHCT seems to be associated with significantly lower rates of cGVHD compared with MUD transplantations.

Thus far, there is only one nonrandomized study that compared HAPLO transplantations with SIB and MUD in patients with HL. ${ }^{25}$ In that retrospective study by Burroughs et al, 90 patients with HL were treated with a nonmyeloablative conditioning regimen followed by alloHCT from an SIB $(n=38)$, an MUD $(n=24)$, or a HAPLO $(n=28)$ donor. HAPLO transplantation was performed by using unmanipulated bone marrow as a graft source and PTCy as GVHD prophylaxis. The authors did not find significant differences in OS among the three groups; however, significantly improved PFS was observed for HAPLO compared with SIB and MUD. NRM and relapse were lowest among HAPLO recipients. In our study, HAPLO compares favorably with SIB in terms of relapse rate and without differences in OS. In contrast to this series, we found no significant differences in post-transplantation outcomes between HAPLO and MUD, except for a lower incidence of cGVHD for HAPLO transplantation. It should be noted that in the study by Burroughs et al, NRM reported after MUD transplantation was unexpectedly low (8\%). We found a 2 -year NRM of $13 \%$ and $23 \%$ after SIB and MUD alloHCT, respectively, which are in line with previous nonrandomized studies that compared SIB and MUD transplantations in patients with HL. ${ }^{3,5,26,27}$ In the current study, 2-year OS (71\% and 62\% after SIB and MUD, respectively) and 2-year PFS (38\% and $45 \%$ after SIB and MUD, respectively) also compare similarly with those reported by other groups. ${ }^{3,5,21,26-28}$

Recently, two retrospective studies from the Center for International Blood and Marrow Transplant Research compared outcomes after HAPLO with MUD and SIB transplantations. ${ }^{18,19}$ Only $22 \%$ of patients who were included in these large series had HL. Both studies- the first comparing HAPLO with MUD with and without ATG, and the second comparing HAPLO with SIB-reported similar survival outcomes with a lower risk of cGVHD for those patients who received HAPLO.

In the current study, no differences between groups in the incidence of acute grade II to IV GVHD were observed; however, the 1-year cumulative incidence of cGVHD was higher for MUD (41\%) compared with SIB $(25 \% ; P=.012)$ and HAPLO $(26 \%$; $P=.04)$. The lower cGVHD rate with HAPLO is in line with recent reports in patients with lymphoma and acute myeloid leukemia. The incidence of acute and CGVHD in our study seems to be lower for all groups than that reported by Burroughs et $\mathrm{al}^{25}$ despite the fact that most patients in our study received PBSC. This could be explained by the use of ATG or alemtuzumab in a significant proportion of patients in the SIB (14\% and $23 \%$, respectively) and MUD (51\% and 23\%, respectively) groups. In our study, the low incidence of cGVHD after HAPLO resulted in a lower rate of cGFRS at 2 years $(40 \%)$ compared with SIB $(28 \% ; P=.049)$ and similar to MUD (38\%; $P=.59)$.

In support of the role of HAPLO for the treatment of relapsed $\mathrm{HL}$, three recently published series report encouraging results. ${ }^{28-30}$ In a retrospective study, Raiola $\mathrm{et}^{\mathrm{al}^{28}}$ reproduced the Burroughs et al data, which confirmed the reproducibility of the PTCy HAPLO 
procedure in terms of toxicity and efficacy. In this series, the cumulative incidence of NRM was low (4\%) and the relapse rate (31\%) was the major cause of treatment failure. Three-year OS and PFS were $77 \%$ and $63 \%$, respectively, in this small series of 26 patients. Gayoso et $\mathrm{al}^{29}$ reported a series of 43 patients who received HAPLO with an RIC approach that substituted total-body irradiation with busulfan in the conditioning regimen in an attempt to reduce the relapse rate. In contrast to the study by Raiola et al, most patients received PBSC graft instead of BMSC. NRM at 1 year (21\%) was similar to that observed in our study (17\%) and higher than that previously reported, likely as a result of the use of more intensive conditioning regimens. ${ }^{21,25}$ Relapse was also the main problem in this series, with a cumulative incidence of $24 \%$ at 2 years. Estimated 2-year PFS and OS were $48 \%$ and 58\%, respectively, and these results compare similarly with our experience. Finally, Castagna et $\mathrm{al}^{30}$ have recently reported similar results (3-year OS, 63\%; and PFS, 59\%) in a series of 62 patients with HL.

This study has some inherent limitations as with other registry-based analyses. The decision to perform an MUD versus a HAPLO transplantation was not an intent-to-treat one; a shorter time from diagnosis to alloHCT for the HAPLO group may suggest that some patients have been offered HAPLO on the basis of institutional preferences, but it may also indicate a shorter time to make the necessary arrangements to perform HAPLO transplantation compared with MUD. Second, the nature of the data captured by the registry does not allow us to evaluate the effect of different types of conditioning regimens on transplant outcome. Thus, although a significantly higher proportion of HAPLO transplantations were performed using RIC regimens compared with SIB and MUD, recent data indicate that, at least for those alloHCTs performed more recently, outcomes after transplantation are similar after myeloablative conditioning regimens and RIC. ${ }^{31}$ Finally, differences in GVHD prophylaxis approaches between SIB and MUD, and the use of BMSC for most HAPLO transplantations versus PBSC for most SIB and MUD transplantations, prevents us from being able to segregate the effect of donor versus GVHD prophylaxis and type of graft source. Nevertheless, this is the largest reported series that compared HAPLO, SIB, and MUD in HL; all HAPLO transplantations were performed by using PTCy as GVHD prophylaxis; and median follow-up for HAPLO transplantations was similar to SIB and MUD.
In the absence of randomized data, our study suggests that PTCy-based HAPLO alloHCT is associated with comparable results with respect to NRM, OS, and PFS with those of conventional transplantations. Moreover, our results indicate that HAPLO results in a lower incidence of extensive cGVHD compared with MUD transplantations and higher cGFRS compared with SIB. Despite the lower cGVHD incidence in the HAPLO group, the risk of relapse was lower than that in the SIB group and similar to that for MUD transplantation group, which suggests that the graftversus-lymphoma effect in the HAPLO setting might be independent of a clinically significant cGVHD. These results support the use of HAPLO as an alternative for patients with advanced HL who lack an SIB donor. Use of HAPLO donors may allow patients to proceed more rapidly to transplantation, avoiding the time needed to complete a formal MUD search and arrange for graft collection at a remote center. Whether HAPLO transplantation is the first choice instead of MUD transplantation and whether it can eventually substitute SIB transplantation in specific subgroups of patients must be assessed within the context of a randomized prospective clinical trial.

\section{AUTHORS' DISCLOSURES OF POTENTIAL CONFLICTS OF INTEREST}

Disclosures provided by the authors are available with this article at jco.org.

\section{AUTHOR CONTRIBUTIONS}

Conception and design: Carmen Martínez, Silvia Montoto, Peter Dreger, Anna Sureda

Provision of study materials or patients: Carmen Martínez

Collection and assembly of data: Jorge Gayoso, Hervé Finel, Karl Peggs, Alida Dominietto, Luca Castagna, Boris Afanasyev, Stephen Robinson, Didier Blaise, Paolo Corradini, Maija Itälä-Remes, Arancha Bermúdez, Edouard Forcade, Domenico Russo, Michael Potter, Grant McQuaker, Ibrahim Yakoub-Agha, Christof Scheid, Adrian Bloor

Data analysis and interpretation: Carmen Martínez, Carmen Canals Manuscript writing: All authors

Final approval of manuscript: All authors

Accountable for all aspects of the work: All authors

\section{REFERENCES}

1. Sureda A, Pereira MI, Dreger $P$, et al: The role of hematopoietic stem cell transplantation in the treatment of relapsed/refractory Hodgkin's lymphoma. Curr Opin Oncol 24:727-732, 2012

2. Sureda $A, B$ ader $P$, Cesaro $S$, et al: Indications for allo- and auto-SCT for haematological diseases, solid tumours and immune disorders: current practice in Europe, 2015. Bone Marrow Transplant 50: 1037-1056, 2015

3. Peggs KS, Hunter $A$, Chopra $R$, et al: Clinical evidence of a graft-versus-Hodgkin's-lymphoma effect after reduced-intensity allogeneic transplantation. Lancet 365:1934-1941, 2005
4. Alvarez I, Sureda A, Caballero MD, et al: Nonmyeloablative stem cell transplantation is an effective therapy for refractory or relapsed Hodgkin lymphoma: Results of a Spanish prospective cooperative protocol. Biol Blood Marrow Transplant 12: 172-183, 2006

5. Anderlini $P$, Saliba $R$, Acholonu $S$, et al: Fludarabine-melphalan as a preparative regimen for reduced-intensity conditioning allogeneic stem cell transplantation in relapsed and refractory Hodgkin's lymphoma: The updated M.D. Anderson Cancer Center experience. Haematologica 93:257-264, 2008

6. Sureda A, Robinson S, Canals $C$, et al: Reduced-intensity conditioning compared with conventional allogeneic stem-cell transplantation in relapsed or refractory Hodgkin's Iymphoma: An analysis from the Lymphoma Working Party of the European Group for Blood and Marrow Transplantation. J Clin Oncol 26:455-462, 2008

7. Robinson SP, Sureda A, Canals $C$, et al: Reduced intensity conditioning allogeneic stem cell transplantation for Hodgkin's lymphoma: Identification of prognostic factors predicting outcome. Haematologica 94:230-238, 2009

8. Sarina B, Castagna L, Farina L, et al: Allogeneic transplantation improves the overall and progressionfree survival of Hodgkin lymphoma patients relapsing after autologous transplantation: A retrospective study based on the time of HLA typing and donor availability. Blood 115:3671-3677, 2010

9. Sureda A, Canals C, Arranz R, et al: Allogeneic stem cell transplantation after reduced intensity conditioning in patients with relapsed or refractory 
Hodgkin's lymphoma. Results of the HDR-ALLO study - A prospective clinical trial by the Grupo Español de Linfomas/Trasplante de Médula Osea (GEL/TAMO) and the Lymphoma Working Party of the European Group for Blood and Marrow Transplantation. Haematologica 97:310-317, 2012

10. Ciurea SO, Mulanovich V, Saliba RM, et al: Improved early outcomes using a $T$ cell replete graft compared with $T$ cell depleted haploidentical hematopoietic stem cell transplantation. Biol Blood Marrow Transplant 18:1835-1844, 2012

11. Aversa F, Tabilio A, Velardi $A$, et al: Treatment of high-risk acute leukemia with T-cell-depleted stem cells from related donors with one fully mismatched HLA haplotype. N Engl J Med 339:1186-1193, 1998

12. Luznik $L, O ' D o n n e l l ~ P V$, Symons $H J$, et al: HLA-haploidentical bone marrow transplantation for hematologic malignancies using nonmyeloablative conditioning and high-dose, posttransplantation cyclophosphamide. Biol Blood Marrow Transplant 14 641-650, 2008

13. Solomon SR, Sizemore CA, Sanacore M, et al: Haploidentical transplantation using $T$ cell replete peripheral blood stem cells and myeloablative conditioning in patients with high-risk hematologic malignancies who lack conventional donors is well tolerated and produces excellent relapse-free survival: Results of a prospective phase II trial. Biol Blood Marrow Transplant 18:1859-1866, 2012

14. Raiola AM, Dominietto $A$, di Grazia $C$, et al: Unmanipulated haploidentical transplants compared with other alternative donors and matched sibling grafts. Biol Blood Marrow Transplant 20:1573-1579, 2014

15. Bashey $A$, Zhang $X$, Sizemore CA, et al: T-cellreplete HLA-haploidentical hematopoietic transplantation for hematologic malignancies using post-transplantation cyclophosphamide results in outcomes equivalent to those of contemporaneous HLA-matched related and unrelated donor transplantation. J Clin Oncol 31:1310-1316 2013

16. Ciurea SO, Zhang MJ, Bacigalupo AA, et al: Haploidentical transplant with posttransplant cyclophosphamide vs matched unrelated donor transplant for acute myeloid leukemia. Blood 126:1033-1040, 2015

17. Castagna L, Bramanti S, Furst S, et al: Nonmyeloablative conditioning, unmanipulated haploidentical SCT and post-infusion CY for advanced lymphomas. Bone Marrow Transplant 49:1475-1480 2014

18. Kanate AS, Mussetti $A$, Kharfan-Dabaja MA et al: Reduced-intensity transplantation for lymphomas using haploidentical related donors vs HLAmatched unrelated donors. Blood 127:938-947, 2016

19. Ghosh N, Karmali R, Rocha V, et al: Reducedintensity transplantation for lymphomas using haploidentical related donors versus HLA-matched sibling donors: A Center for International Blood and Marrow Transplant Research analysis. J Clin Oncol 34:3141-3149, 2016

20. Dietrich S, Finel H, Martinez $C$, et al: Posttransplant cyclophosphamide-based haplo-identica transplantation as alternative to matched sibling or unrelated donor transplantation for non-Hodgkin lymphoma: A registry study by the European Society for Blood and Marrow Transplantation. Leukemia 30:2086-2089, 2016

21. McCurdy SR, Kasamon $Y L$, Kanakry CG, et al: Comparable composite endpoints after HLA-matched and HLA-haploidentical transplantation with posttransplantation cyclophosphamide. Haematologica 102:391-400, 2017

22. Kröger N, Solano C, Wolschke C, et al: Antilymphocyte globulin for prevention of chronic graftversus-host disease. N Engl J Med 374:43-53, 2016

23. Devetten MP, Hari PN, Carreras J, et al: Unrelated donor reduced-intensity allogeneic hematopoietic stem cell transplantation for relapsed and refractory Hodgkin lymphoma. Biol Blood Marrow Transplant 15:109-117, 2009

24. Solomon SR, Sizemore $C$, Ridgeway $M$, et al: Current graft-versus-host disease (GVHD), relapsefree survival-A novel, dynamic composite endpoint to better define effectiveness following allogeneic hematopoietic cell transplantation. Blood 128:1170, 2016 (abstr 1170)

25. Burroughs LM, O'Donnell PV, Sandmaier BM, et al: Comparison of outcomes of HLA-matched related, unrelated, or HLA-haploidentical related hematopoietic cell transplantation following nonmyeloablative conditioning for relapsed or refractory Hodgkin lymphoma. Biol Blood Marrow Transplant 14:1279-1287, 2008

26. Anderlini $P$, Saliba $R$, Acholonu $S$, et al: Reduced-intensity allogeneic stem cell transplantation in relapsed and refractory Hodgkin's disease: Low transplant-related mortality and impact of intensity of conditioning regimen. Bone Marrow Transplant 35 943-951, 2005

27. Armand $P, \mathrm{Kim} H T, H o V T$, et al: Allogeneic transplantation with reduced-intensity conditioning for Hodgkin and non-Hodgkin lymphoma: Importance of histology for outcome. Biol Blood Marrow Transplant 14:418-425, 2008

28. Raiola A, Dominietto $A$, Varaldo $R$, et al: Un manipulated haploidentical BMT following nonmyeloablative conditioning and post-transplantation CY for advanced Hodgkin's lymphoma. Bone Marrow Transplant 49:190-194, 2014

29. Gayoso J, Balsalobre P, Pascual MJ, et al: Busulfan-based reduced intensity conditioning regimens for haploidentical transplantation in relapsed/ refractory Hodgkin lymphoma: Spanish multicenter experience. Bone Marrow Transplant 51:1307-1312, 2016

30. Castagna L, Bramanti S, Devillier R, et al: Haploidentical transplantation with post-infusion cyclophosphamide in advanced Hodgkin lymphoma Bone Marrow Transplant 1:1-6, 2017

31. Genadieva-Stavrik S, Boumendil A, Dreger P et al: Myeloablative versus reduced intensity allogeneic stem cell transplantation for relapsed/refractory Hodgkin's lymphoma in recent years: A retrospective analysis of the Lymphoma Working Party of the European Group for Blood and Marrow Transplantation. Ann Oncol 27:2251-2257, 2016

\section{Affiliations}

Carmen Martínez, Institute of Hematology and Oncology, Hospital Clínic; Carmen Canals, Banc de Sang i Teixits; Anna Sureda, Institut Català d'Oncologia, Hospital Duran i Reynals, Barcelona; Jorge Gayoso, Hospital General Universitario Gregorio Marañón, Madrid; Arancha Bermúdez, Hospital Universitario Marqués de Valdecilla, Cantabria, Spain; Carmen Martínez, Hervé Finel, Silvia Montoto, Peter Dreger, and Anna Sureda, European Society for Blood and Marrow Transplantation, Paris; Didier Blaise, Institut Paoli Calmettes, Marseille; Edouard Forcade, University Hospital of Bordeaux, Bordeaux; Ibrahim Yakoub-Agha, Centre Hospitalier Regional Universitaire de Lille, Lille Inflammation Research International Center, Institut National de la Santé et de la Recherche Médicale U995, Lille, France; Karl Peggs, University College London Cancer Institute; Michael Potter, The Royal Marsden Hospital; Silvia Montoto, St Bartholomew's Hospital, Barts Health National Health Service Trust, London; Stephen Robinson, Bristol Haematology and Oncology Centre, Bristol; Grant McQuaker, Gartnaval General Hospital, Glasgow; Adrian Bloor, Christie National Health Service Trust Hospital, Manchester, United Kingdom; Alida Dominietto, Istituto di Ricovero e Cura a Carattere Scientifico per l'Oncologia, Azienda OspedalieraUniversitaria San Martino-IST, Genova; Luca Castagna, Istituto Clinico Humanitas; Paolo Corradini, Istituto Nazionale dei Tumori, Milan; Domenico Russo, University of Brescia, Brescia, Italy; Boris Afanasyev, First Pavlov State Medical University of St Petersburg, St Petersburg, Russia; Maija Itälä-Remes, Turku University Hospital, Turku, Finland; Christof Scheid, University of Cologne, Cologne; and Peter Dreger, University of Heidelberg, Heidelberg, Germany.

\section{Prior Presentation}

Presented in part at the 57th Annual Meeting of the American Society of Hematology, Orlando, FL, December 5-8, 2015, and the 42nd Annual Meeting of the European Society for Blood and Marrow Transplantation, Valencia, Spain, April 3-6, 2016. 


\section{AUTHORS' DISCLOSURES OF POTENTIAL CONFLICTS OF INTEREST}

Post-Transplantation Cyclophosphamide-Based Haploidentical Transplantation as Alternative to Matched Sibling or Unrelated Donor Transplantation for Hodgkin Lymphoma: A Registry Study of the Lymphoma Working Party of the European Society for Blood and Marrow Transplantation

The following represents disclosure information provided by authors of this manuscript. All relationships are considered compensated. Relationships are self-held unless noted. I = Immediate Family Member, Inst = My Institution. Relationships may not relate to the subject matter of this manuscript. For more information about ASCO's conflict of interest policy, please refer to www.asco.org/rwc or ascopubs.org/jco/site/ifc.

Carmen Martínez

No relationship to disclose

\section{Jorge Gayoso}

Consulting or Advisory Role: Celgene, Takeda, MSD, Roche, AbbVie, Astellas, Amgen, Addiene

Travel, Accommodations, Expenses: Celgene, Janssen Pharmaceuticals

\section{Carmen Canals}

No relationship to disclose

Hervé Finel

No relationship to disclose

\section{Karl Peggs}

Honoraria: Takeda, Merck, Chimerix, Pfizer, Bristol-Myers Squibb Consulting or Advisory Role: Bristol-Myers Squibb, Pfizer, Chimerix, Takeda

Speakers' Bureau: Takeda

Travel, Accommodations, Expenses: Takeda

\section{Alida Dominietto}

No relationship to disclose

\section{Luca Castagna}

No relationship to disclose

\section{Boris Afanasyev}

No relationship to disclose

\section{Stephen Robinson}

Honoraria: Roche, Takeda, Gilead Sciences

Consulting or Advisory Role: Takeda

Travel, Accommodations, Expenses: Roche, Takeda, Gilead Sciences

\section{Didier Blaise}

No relationship to disclose

Paolo Corradini

Honoraria: Celgene, Janssen Pharmaceuticals, Novartis, Takeda, Gilead Sciences

Travel, Accommodations, Expenses: Novartis, Celgene, Roche

Maija Itälä-Remes

No relationship to disclose

Arancha Bermúdez

No relationship to disclose

\section{Edouard Forcade}

Honoraria: Pfizer

Domenico Russo

No relationship to disclose

Michael Potter

Honoraria: MSD

Consulting or Advisory Role: MSD

Grant McQuaker

Honoraria: Celgene, Jazz Pharmaceuticals, Takeda

Consulting or Advisory Role: Takeda

Travel, Accommodations, Expenses: Celgene, Takeda

Ibrahim Yakoub-Agha

No relationship to disclose

Christof Scheid

Honoraria: Amgen, Bristol-Myers Squibb, Celgene, Janssen Pharmaceuticals, Novartis

Consulting or Advisory Role: Amgen, Bristol-Myers Squibb, Novartis Travel, Accommodations, Expenses: Amgen, Bristol-Myers Squibb, Celgene, Novartis

\section{Adrian Bloor}

Leadership: The Doctors Laboratory

Honoraria: AbbVie

Consulting or Advisory Role: AbbVie, Roche

Speakers' Bureau: Janssen Pharmaceuticals

Travel, Accommodations, Expenses: AbbVie, Gilead Sciences

Silvia Montoto

Consulting or Advisory Role: Bayer

Peter Dreger

Honoraria: Novartis, Gilead Sciences

Consulting or Advisory Role: Janssen Oncology, Gilead Sciences, Roche (Inst), Novartis (Inst)

Research Funding: Neovii Biotech (Inst), RIEMSER (Inst)

Travel, Accommodations, Expenses: Medac

Anna Sureda

No relationship to disclose 


\section{Acknowledgment}

We acknowledge the kind support of all physicians and data managers of the participating transplantation centers.

\section{Appendix}

\section{Hematopoietic Recovery}

Median time to an absolute neutrophil count of $\geq 500 / \mathrm{mm}^{3}$ for patients who received bone marrow stem-cells was 18 days for haploidentical (HAPLO), 20 days for sibling (SIB), and 20 days for HLA-matched unrelated donor (MUD; $P=.23$ ) transplantation. Platelet recovery was not significantly different among groups (median time to a platelet count of $\geq 20,000 / \mathrm{mm}^{3}$ was 20,18 , and 16 days for HAPLO, SIB, and MUD, respectively; $P=.45$ ). For those patients who received peripheral blood stem-cell transplantation, median time for absolute neutrophil count of $\geq 500 / \mathrm{mm}^{3}$ was 18,14 , and 14 days for HAPLO, SIB, and MUD, respectively $(P<.001)$. Platelet recovery was significantly faster for SIB and MUD recipients compared with patients in the HAPLO group (13 and 12 days $v 25$ days, respectively; $P<.001$ ).

\section{Effect of Antithymocyte Globulin or Campath Use on SIB and MUD alloHCT Outcomes}

Because there was a tight correlation between donor type and the type of graft-versus-host-disease prophylaxis, we performed a subset analysis of allogeneic hematopoietic cell transplantation (alloHCT) outcomes to assess the potential effect of antithymocyte globulin (ATG) or campath on SIB and MUD alloHCT outcomes. Two separate multivariable analyses were performed, one that was restricted to SIB with and without ATG or campath and another restricted to MUD with and without ATG or campath. Use of ATG or campath did not have any effect on nonrelapse mortality (NRM), relapse, overall survival (OS), or progression-free survival (PFS) in the SIB setting. Whereas the use of campath did not have any effect on alloHCToutcomes in the MUD group, use of ATG was associated with a lower PFS (hazard ratio, $1.51 ; 95 \% \mathrm{CI}, 1.1$ to $2.1 ; P=.02$ ), with no differences in the risk of NRM, relapse, and OS. A subsequent multivariable analysis that included HAPLO, SIB, MUD with ATG, and MUD without ATG indicated that HAPLO was associated with a lower relapse rate than SIB (hazard ratio, $0.69 ; 95 \%$ CI, 0.48 to $1.0 ; P=.047$ ) with no significant differences in NRM, OS, or PFS (Appendix Table A1). No differences were observed between HAPLO and MUD with or without ATG. These results were in line with the outcomes shown in the main analysis. 
Table A1. Multivariable Cox Proportional Hazards Models With Donor Type as a Covariate: Analysis of Four Groups (HAPLO, SIB, MUD with ATG, and MUD without ATG)

\begin{tabular}{|c|c|c|c|}
\hline Factor & $\mathrm{HR}$ & $95 \% \mathrm{Cl}$ & $P$ \\
\hline \multicolumn{4}{|l|}{ Cox model on NRM } \\
\hline \multicolumn{4}{|l|}{ Donor type } \\
\hline \multicolumn{4}{|l|}{ SIB } \\
\hline HAPLO & 1.38 & 0.79 to 2.39 & .25 \\
\hline MUD with ATG & 2.31 & 1.50 to 3.54 & $<.001$ \\
\hline MUD without ATG & 1.31 & 0.81 to 2.12 & .28 \\
\hline \multicolumn{4}{|l|}{ Age, years } \\
\hline \multicolumn{4}{|l|}{$\leq 39$} \\
\hline$\geq 40$ & 1.83 & 1.28 to 2.63 & $<.001$ \\
\hline \multicolumn{4}{|l|}{ Performance status } \\
\hline \multicolumn{4}{|l|}{ Good } \\
\hline Poor & 2.39 & 1.22 to 4.68 & .011 \\
\hline \multicolumn{4}{|l|}{ Refractory HL } \\
\hline \multicolumn{4}{|l|}{ Nonrefractory } \\
\hline Refractory & 1.60 & 1.03 to 2.47 & .037 \\
\hline \multicolumn{4}{|c|}{ Cox model on relapse rate } \\
\hline \multicolumn{4}{|l|}{ Donor type } \\
\hline \multicolumn{4}{|l|}{ SIB } \\
\hline HAPLO & 0.69 & 0.48 to 1.0 & .047 \\
\hline MUD with ATG & 0.67 & 0.48 to 0.94 & .019 \\
\hline MUD without ATG & 0.48 & 0.34 to 0.68 & $<.001$ \\
\hline \multicolumn{4}{|l|}{ Sex } \\
\hline \multicolumn{4}{|l|}{ Female } \\
\hline Male & 1.28 & 1.01 to 1.64 & .045 \\
\hline \multicolumn{4}{|l|}{ Performance status } \\
\hline \multicolumn{4}{|l|}{ Good } \\
\hline Poor & 1.84 & 1.08 to 3.13 & .026 \\
\hline \multicolumn{4}{|l|}{ Refractory HL } \\
\hline Nonrefractory & & & \\
\hline Refractory & 2.68 & 2.98 to 6.37 & $<.001$ \\
\hline Cox model on OS & & & \\
\hline Donor type & & & \\
\hline SIB & & & \\
\hline HAPLO & 1.27 & 0.86 to 1.86 & .22 \\
\hline MUD with ATG & 1.71 & 1.25 to 2.36 & .001 \\
\hline MUD without ATG & 1.27 & 0.91 to 1.78 & .16 \\
\hline Age, years & & & \\
\hline$\leq 39$ & & & \\
\hline$\geq 40$ & 1.58 & 1.22 to 2.06 & .001 \\
\hline Performance status & & & \\
\hline Good & & & \\
\hline Poor & 2.37 & 1.48 to 3.80 & $<.001$ \\
\hline Refractory HL & & & \\
\hline Nonrefractory & & & \\
\hline Refractory & 2.13 & 1.61 to 2.83 & $<.001$ \\
\hline Cox model on PFS & & & \\
\hline Donor type & & & \\
\hline SIB & & & \\
\hline HAPLO & 0.87 & 0.64 to 1.17 & .36 \\
\hline MUD with ATG & 1.03 & 0.80 to 1.33 & .80 \\
\hline MUD without ATG & 0.67 & 0.51 to 0.89 & .005 \\
\hline Age, years & & & \\
\hline$\leq 39$ & & & \\
\hline$\geq 40$ & 1.29 & 1.04 to 1.60 & .021 \\
\hline Performance status & & & \\
\hline Good & & & \\
\hline Poor & 2.0 & 1.32 to 3.03 & .001 \\
\hline Refractory HL & & & \\
\hline Nonrefractory & & & \\
\hline Refractory & 2.22 & 1.76 to 2.80 & $<.001$ \\
\hline
\end{tabular}

Abbreviations: ATG, antithymocyte globulin; HAPLO, haploidentical donor; HL, Hodgkin lymphoma; HR, hazard ratio; MUD, match-unrelated donor; NRM, nonrelapse mortality; OS, overall survival; PFS, progression-free survival; SIB, sibling donor. 